\title{
Optimal Configuration of Wind Turbine and Photovoltaic Based on Day-ahead Economic Dispatch
}

\author{
Bing Sun*, Yunfei Li, Yuan Zeng \\ Key Laboratory of Smart Grid of Ministry of Education, Tianjin University, Tianjin, China \\ Email address: \\ sunbing@tju.edu.cn (Bing Sun), 15822085262@163.com (Yunfei Li), zengyuan@tju.edu.cn (Yuan Zeng) \\ ${ }^{*}$ Corresponding author
}

\section{To cite this article:}

Bing Sun, Yunfei Li, Yuan Zeng. Optimal Configuration of Wind Turbine and Photovoltaic Based on Day-ahead Economic Dispatch. Science Discovery. Vol. 9, No. 3, 2021, pp. 91-96. doi: 10.11648/j.sd.20210903.11

Received: March 8, 2021; Accepted: April 24, 2021; Published: May 8, 2021

\begin{abstract}
With the gradual increase of the penetration rate of wind and photovoltaic (PV) electricity, the power system is facing great challenges in integration and peak-shaving capacity. As flexibility resources of the system is limited, wind and PV power generation will not be consumed completely. Allowing the curtailment of the peak output of wind turbine and PV can reduce the cost of auxiliary services such as peak-shaving, relieve the huge pressure in the operation and planning of the system, and improve the overall power supply economy of the system. Firstly, the reason and optimization principle of the curtailment of the peak output of wind turbine and PV are explained. Then, a capacity optimal configuration method of power supply based on day-ahead economic dispatch is proposed. On the premise of allowing the curtailment of the peak output of wind turbine and PV, the corresponding annual power supply cost of wind turbine and PV capacity combination is calculated based on day-ahead unit commitment. The function fitting between power supply cost and wind turbine capacity is carried out. The best wind turbine and PV capacity combination is the value of the extreme point. Finally, the sensitivity analysis of example system is carried out. It is found that with the decrease of unit investment cost of wind turbine and PV equipment, developing wind and PV power generation will be a more economical choice in the future.
\end{abstract}

Keywords: Economic Dispatch, High-penetration Power System, Economic Consumption of Electricity, Curtailment Ratio of Electricity, Unit Electricity Supply Cost, Capacity Configuration Planning

\section{基于日前经济调度的风光机组容量优化配置方法}

孙冰 ${ }^{*}$, 李云飞, 曾沅

教育部智能电网重点实验室, 天津大学, 天津, 中国

邮箱

sunbing@tju.edu.cn（孙冰），15822085262@163.com（李云飞）, zengyuan@tju.edu.cn（曾沅）

\begin{abstract}
摘要：随着风光电量渗透率的逐步提高, 电力系统在电网接纳能力、调峰能力等方面面临巨大的挑战, 由于系统 灵活性资源有限, 将不能 $100 \%$ 地消纳风光电能, 允许弃置一部分风光机组尖峰出力, 可以降低系统调峰等辅助服 务的成本，缓解系统运行和规划中面临的巨大压力，同时提高系统整体供电经济性。首先，通过一个简化的电力 系统阐释了弃风弃光现象的原因和优化原理; 然后, 提出了一种基于日前经济调度的电源容量优化配置方法, 以 允许弃置一部分风光机组尖峰出力为前提, 基于时序运行仿真求取风光机组装机容量组合的年供电成本, 进而对 多组年供电成本与风光机组装机容量开展函数拟合, 在极值点处获得经济性最佳的风光机组容量规划方案; 最后, 对给定场景的系统开展了敏感性分析, 发现随着风光机组单位投资成本的下降, 大力发展风光发电将会是未来更 为经济的选择。
\end{abstract}


关键词: 经济调度, 高渗透率电力系统, 电能经济消纳, 弃风弃光比例, 单位电能供电成本, 容量规划

\section{1. 引言}

为了应对环境污染、气候问题和实现可持续发展，世 界各国都在大力发展风电和光伏发电, 我国风机和光伏的 累计装机容量均为世界第一[1-2]。随着科学技术的不断进 步，风机、光伏的设备成本也呈现出快速下降的趋势，但 是，也应该认识到风、光机组的大规模并网使得电力系统 面临调峰、备用等多方面的挑战。

风光机组发电具有间歇性、多变性以及不确定性[3-4], 必须在其他出力可控机组的配合下才能向负荷稳定供电。 随着风光电量渗透率的逐步提高, 由于系统灵活性资源有 限, 将不能 $100 \%$ 地消纳风光电能, 必须考虑风光电能的 经济消纳, 即: 通过合理弃置一部分风光机组的尖峰出力, 缓解系统运行和规划中面临的巨大压力, 同时提高系统整 体供电经济性。

针对风光机组装机容量的优化问题, 已有大量的研究, 所建模型以及求解算法上面各有侧重。由于可再生能源之 间存在天然的互补性，与仅使用风机或仅使用光伏的情况 相比, 风-光混合系统供电具有较低的供电成本 [5]; 将强 度Pareto进化算法应用于孤立混合系统的多目标设计中, 可以使系统在整个使用寿命期内的总费用和未满足的载 荷最小化[6]; 在大型绿色船舶的混合能源系统中, 通过采 用多种能源来满足电力需求, 可以在节省大量电费和强大 控制性能的情况下获得被评估系统的最佳能源管理[7]; 停 电将会给用户带来巨大的损失, 因此在电源容量优化模型 中还需计及可靠性成本 [8]。已有文献大多从经济性角度出 发通过不同的智能优化算法对所建立模型可再生能源的 装机容量进行了优化, 但是忽略了随着风机和光伏的大量 接入, 电力系统内存在的过发电现象, 因此系统必须提供 更多的辅助服务来保证系统的灵活性和供电可靠性, 而所 需要的辅助服务的成本以及作为调峰机组所产生的 $\mathrm{CO}_{2}$ 等 废气对环境的影响也应该计入总成本 [9-11], 并且应该考 虑治理环境所需要的成本。

随着风光电量渗透率的增加, 对于灵活性资源的需求 也逐步凸显, 当电源组合中存在间歇性可再生能源时, 存 储技术是为电力系统提供所需灵活性的一个很有希望的 选择 [12], 但就目前而言, 储能因为其较高的投资成本很 难大规模使用。允许弃置一定比例的风光机组尖峰出力不 仅可以提高可再生能源的渗透率 [13], 还可以防止由于可 再生能源出力的变动性而导致火电机组无法短时间内立 刻启动导致的切负荷行为 [14]。通过研究可再生能源的间 歇性出力对供电成本的影响, 舍弃可再生能源的部分出力 可以提高系统的备用容量, 是系统变得更加安全可靠 [15], 并且当系统灵活性较低而可再生能源变动比较剧烈的时 候, 舍弃部分可再生能源出力也是更为经济的选择 [16], 如果一味的减少可再生能源的弃置电量去保证可再生能 源的 $100 \%$ 消纳, 系统运行成本将大幅度提升 $[17$ 。在现有 研究中也并未将风机和光伏的装机成本考虑在内, 也没有 更加深入研究包括系统中火电机组的运行成本以及可再
生能源的装机成本在内的系统总成本与舍弃的可再生能 源电量之间的关系, 从而无法确定使得供电经济性最佳的 风光机组装机容量组合。

针对已有研究的不足, 本文提出了一种基于日前经济 调度的风光机组装机容量的优化配置方法, 其核心是基于 运行仿真寻找使得总供电成本最小的风光装机容量及对 应的弃风弃光比例。首先, 通过一个简化的电力系统阐释 了弃风弃光现象的原因和优化原理; 然后, 建立了风光机 组装机容量的数学优化模型, 并给出了模型的有效求解方 法; 最后, 对给定场景的系统开展了敏感性分析, 发现随 着风光机组单位投资成本的下降, 大力发展风光机组将会 是未来更为经济的选择。

\section{2. 风光电能经济消纳}

对于选定的风机光伏装机容量组合, 弃风弃光比例和 单位电能供电成本分别按式 (1) 和式 (2) 计算:

$$
\begin{gathered}
R_{\mathrm{rej}}\left(P_{\mathrm{PV}}, P_{\mathrm{wind}}\right)=\frac{E_{1}\left(P_{\mathrm{PV}}, P_{\mathrm{wind}}\right)-E_{2}\left(P_{\mathrm{PV}}, P_{\mathrm{wind}}\right)}{E_{1}\left(P_{\mathrm{PV}}, P_{\text {wind }}\right)} \\
C_{\mathrm{kWh}}\left(P_{\mathrm{PV}}, P_{\text {wind }}\right)=\frac{\sum\left(C_{1}+C_{2}+C_{3}\right)}{E_{\text {year }}}
\end{gathered}
$$

其中, $E_{1}\left(P_{\mathrm{PV}}, P_{\text {wind }}\right)$ 表示光伏和风机装机容量为 $\left(P_{\mathrm{PV}}, P_{\text {wind }}\right)$ 时, 充分利用当地的风光资源风光机组每年可 发出的最大电量; $E_{2}\left(P_{\mathrm{PV}}, P_{\text {wind }}\right)$ 表示由于运行需要, 实际 的年发电量; $C_{1} 、 C_{2} 、 C_{3}$ 分别表示系统投资成本、燃料成 本、环境成本，它们的计算方法见3.1节; $E_{\text {year }}$ 表示每年 系统终端负荷的用电量。

通常, 期望电力系统能够最大限度乃至 $100 \%$ 地消纳 风光电能, 出现弃风弃光是一件不好的事情, 这种认知惯 性导致对风光电能经济消纳的关注不足。下面结合图1对 风光电能经济消纳进行解释: 对某既定的电力系统, 当并 网风光机组容量较小时, 能够实现风光电量的 $100 \%$ 消纳, 清洁的风光电能替代了部分传统电能, 往往能够降低系统 单位电能供电成本; 随着风光机组并网容量的逐步增加, 风光机组的尖峰出力将不能被消纳, 开始出现弃风弃光现 象, 系统的单位电能供电成本下降速度变缓, 对应图1中 的 $\mathrm{D}_{1}$; 继续增加并网风光机组, 会使得弃风弃光电量进一 步增多, 弃风弃光比例升高, 并最终使得系统供电经济性 出现拐点, 此时系统的单位电能供电成本达到最低, 弃风 弃光措施与系统灵活资源之间实现最优配合, 对应图1中 的 $\mathrm{D}_{2}$; 如果继续向该系统中增加并网风光机组, 会导致弃 风弃光比例进一步升高, 由于大量风光设备闲置而使得系 统单位电能供电成本上升, 对应图1中的 $\mathrm{D}_{3}$ 。由此可见, 从供电经济性的角度, 系统能够接纳的并网风光机组存在 
某个最优容量, 此时风光电量渗透率达到最佳数值, 弃风 弃光比例也达到最佳数值。

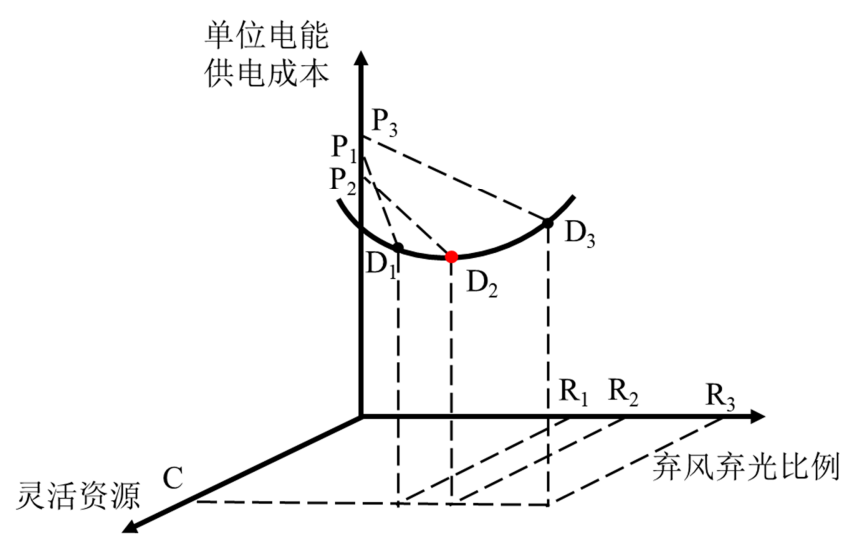

图1 风光电能经济消纳示意图。

以单机系统为例, 利用一台额定容量为 $1000 \mathrm{MW}$ 的超 超临界机组向 $900 \mathrm{MW}$ 的恒定负荷供电, 风机和光伏装机 容量的比值为 $1: 1$ 时, 火电机组的煤耗成本与风机/光伏装 机容量之间的函数关系如图 2 所示, 此时, 系统中最佳风 机/光伏装机容量为 $550 \mathrm{MW}$, 当系统中存在多台火电机组 时, 则需要开展日前经济调度确定最佳风光机组装机容量 组合。

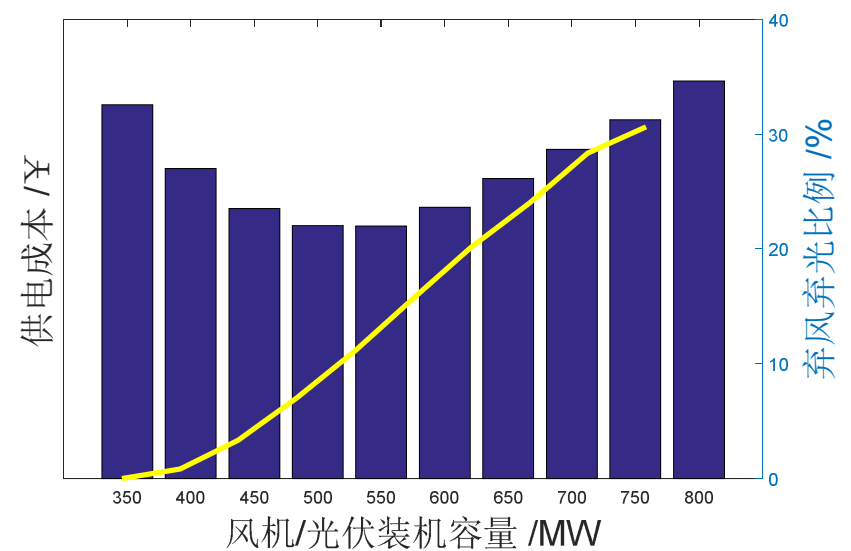

图2 单机系统供电成本与风机/光伏装机容量之间的关系。

\section{3. 风光电能经济消纳模型及求解方法}

\section{1. 系统成本构成}

对于典型的风-光-火互补发电系统的供电成本主要包 括: (1)风、光、火机组的设备投资及维护费用, 简称为系 统的投资成本, (2)火电机组的运行成本, (3)环境成本, (4) 调峰和备用成本, 其中调峰和备用成本是由火电机组提供 的, 已经隐性地包含在前两项成本中, 本文不再单独计及。

(1) 系统的投资成本

采用等年值方法计及互补系统的年运行成本, 其中等 年值系数 $c$ 为:
其中, $r$ 表示银行贴现率, $x$ 表示设备的运行年限。三 类机组的设备投资及维护费用记为 $C_{1}$, 可通过下式计算:

$$
\begin{aligned}
& C_{1}=\left(c_{\mathrm{w}}+c_{\text {om_w }}\right) C_{\text {un_w } \mathrm{w}} P_{\mathrm{w}}+\left(c_{\mathrm{PV}}+c_{\mathrm{om} \_\mathrm{PV}}\right) C_{\mathrm{un} \_\mathrm{PV}} P_{\mathrm{PV}} \\
& +\left(c_{\mathrm{f}}+c_{\text {om_f }}\right)\left(C_{\mathrm{un} \_ \text {ul }} N_{\mathrm{ul}} P_{\mathrm{ul}}+C_{\mathrm{un} \_ \text {su }} N_{\mathrm{su}} P_{\mathrm{su}}\right)
\end{aligned}
$$

其中, $c_{\mathrm{W}} 、 c_{\mathrm{PV}}$ 和 $c_{\mathrm{f}}$ 分别表示风机、光伏和火电机组的

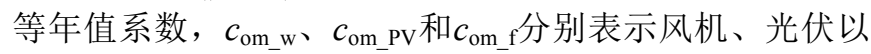
及火电机组的运维比例 [4], $C_{\mathrm{un} \_\mathrm{w}} 、 C_{\mathrm{un} \_\mathrm{PV}} 、 C_{\mathrm{un} \_\mathrm{ul}}$ 和 $C_{\mathrm{un} \_ \text {su }}$ 分别表示风机、光伏、超超临界火电和超临界火电机组的 单位容量投资成本, $P_{\mathrm{ul}}$ 和 $P_{\mathrm{su}}$ 分别表示超超临界火电和超 临界火电机组的额定容量, $N_{\mathrm{ul}}$ 和 $N_{\mathrm{su}}$ 分别表示超超临界和 超临界机组的数量。

(2) 火电机组的运行成本

火电机组的运行成本包括燃料成本和启停成本, 其中 燃料成本是指所消耗一次能源 (如煤炭) 的购买成本。运 行成本 $C_{2}$ 可采用如下计算公式。

$$
C_{2}=\sum_{i=1}^{N_{\mathrm{un}}+N_{\mathrm{su}} 8760} \sum_{t=1} S_{i, t} C_{\text {coal }}\left(a_{i} P_{i, t}+b_{i}\right)+\sum_{i=1}^{N_{\mathrm{un}}+N_{\mathrm{su}} 87600} \sum_{t=1} C_{\mathrm{up}, i} S_{i, t}\left(1-S_{i, t-1}\right)
$$

其中, $C_{\text {coal }}$ 表示煤炭的价格; $a_{i} 、 b_{i}$ 为火电机组燃料 消耗系数; $S_{i, 1}$ 表示机组的启停状态, 为 $0-1$ 变量, 0 代表关 机, 1 代表开机; $C_{\mathrm{up}, i}$ 为第 $i$ 台机组的单次启动成本; $P_{i}, t$ 表示第 $i$ 台火电机组 $t$ 时刻的有功功率。具体参数见文献[4], 不再展开。

(3) 环境成本

环境成本包括污染物的排放成本和治理成本以及 $\mathrm{CO}_{2}$ 的排放成本, 该项成本记为 $C_{3}$, 可通过每吨标煤的环境成 本与燃煤总量的乘积得到, 相关参数见文献[4]。

$$
C_{3}=\sum_{i=1}^{N_{\mathrm{ul}}+N_{\mathrm{su}}} \sum_{t=1}^{8760} S_{i, t} C_{\mathrm{en}}\left(a_{i} P_{i, t}+b_{i}\right)
$$

其中, $C_{\mathrm{en}}$ 表示每吨标煤的环境成本。

\section{2. 数学模型}

规划模型的决策变量是 $P_{\mathrm{PV}}$ 和 $P_{\mathrm{w}}$, 以互补电源系统的 年供电成本最小为目标函数确定 $P_{\mathrm{PV}}$ 和 $P_{\mathrm{w}}$ :

$$
f\left(P_{\mathrm{PV}}, P_{\mathrm{w}}\right)=\min \left(C_{1}+C_{2}+C_{3}\right)
$$

为了求解 $C_{2}$ 和 $C_{3}$, 需要确定 $P_{i, t}$ 本文以 24 小时为一个 周期, 采用日前机组组合运行优化模型, 求解日运行成本 最小的火电机组时序出力 $P_{i, t}$, 需要开展一年 365 天的连续 日前经济调度仿真, 通过仿真来确定全年的风光机组和火 电机组时序出力。

$$
\min \sum_{t=1}^{24} C_{2}
$$

$$
c=\frac{r(1+r)^{x}}{(1+r)^{x}-1}
$$


其中, 式 (8) 在优化过程中要满足风-光-火互补电源 系统要满足系统功率平衡约束:

$$
\begin{aligned}
& P_{\mathrm{w}, t}+P_{\mathrm{PV}, t}+\sum_{i=1}^{N_{\mathrm{ul}}+N_{\mathrm{su}}} P_{i, t}=P_{l, t} \\
& \forall \quad t \in[1,2, \cdots, 8760]
\end{aligned}
$$

火电机组出力要满足最小/最大出力约束、最大爬坡 速率约束、最小开机/停机时长约束:

$$
\begin{aligned}
& \left\{\begin{array}{l}
S_{i, t} P_{i, \text { min }} \leq P_{i, t} \leq S_{i, t} P_{i, \text { max }} \\
-P_{i, \text { ram }} \leq P_{i, t}-P_{i, t-1} \leq P_{i, \text { ram }} \\
T_{i, \text { minon }} \leq T_{i, t, \text { on }} \\
T_{i, \text { minoff }} \leq T_{i, t \text { off }}
\end{array}\right. \\
& \forall \quad i \in\left[1,2, \cdots, N_{\mathrm{ul}}+N_{\text {su }}\right], t \in[1,2, \cdots, 8760]
\end{aligned}
$$

其中, $P_{i, \text { min }}$ 和 $P_{i, \text { max }}$ 分别表示第 $i$ 台火电机组的技术最 小出力和最大出力; $P_{i, \text { ram }}$ 表示第 $i$ 台机组的最大爬坡功率; $T_{i, \text { minon }}$ 和 $T_{i, \text { minoff }}$ 分别表示第 $i$ 台机组的最小开机和最小停机 时长; $T_{i, t, \mathrm{on}}$ 和 $T_{i, t, \mathrm{off}}$ 分别表示第 $i$ 台机组 $t$ 时刻的已经持续的 开机和停机时长。

火电机组出力要存在上、下备用容量, 以应对风光机 组出力预测误差和负荷预测值误差:

$$
\begin{aligned}
& \left\{\begin{array}{l}
\sum_{i=1}^{N_{\mathrm{ul}}+N_{\mathrm{su}}}\left(P_{i, t}-S_{i, t} P_{i, \text { min }}\right) \geq \alpha\left(P_{\mathrm{PV}, t}+P_{\mathrm{w}, t}\right) \\
\sum_{i=1}^{N_{\mathrm{u}}+N_{\mathrm{su}}}\left(S_{i, t} P_{i, \text { max }}-P_{i, t}\right) \geq \beta P_{\mathrm{l}, t}+\alpha\left(P_{\mathrm{PV}, t}+P_{\mathrm{w}, t}\right)
\end{array}\right. \\
& \forall t \in[1,2, \cdots, 8760]
\end{aligned}
$$

其中, $\alpha$ 和 $\beta$ 分别表示与风光机组出力和负荷功率相对 应的备用系数。

\section{3. 模型求解方法}

弃风弃光比例是风机和光伏装机容量的函数, 因此应 该对风机和光伏装机容量组成的装机容量的二维平面进 行遍历去寻得年供电成本的最小值, 本文提出一种简化思 路, 采用线优化思路快速求解优化规划模型。

首先, 把风机和光伏机组的容量之比记为 $k_{\mathrm{c}}$, 在以 $\left(P_{\mathrm{PV}}\right.$, $\left.P_{\mathrm{w}}\right)$ 为变量的二维平面上, 确定 $N$ 个 $k_{\mathrm{c}}$ 取值所对应的 $\left(P_{\mathrm{PV}}, P_{\mathrm{w}}\right)$ 射线, 如图3所示; 然后, 在每条射线上确定 $m$ 个 $R_{\mathrm{rej}}$ 属于 $\left[\begin{array}{ll}0 & R\end{array}\right]$ 的 $\left(P_{\mathrm{PV}}, P_{\mathrm{w}}\right)$, 对每一个 $\left(P_{\mathrm{PV}}, P_{\mathrm{w}}\right)$ 通过日前经济调度确 定各台机组的时序出力曲线; 最后, 对不同的 $\left(P_{\mathrm{PV}}, P_{\mathrm{w}}\right)$ 方 案开展成本敏感度分析, 确定最佳风光机组容量规划方案。 通过线优化思路, 可以加快求解速度。风机光伏装机容量 配比为 $k_{\mathrm{c}}$ 时优化模型的求解流程如图 4 所示。

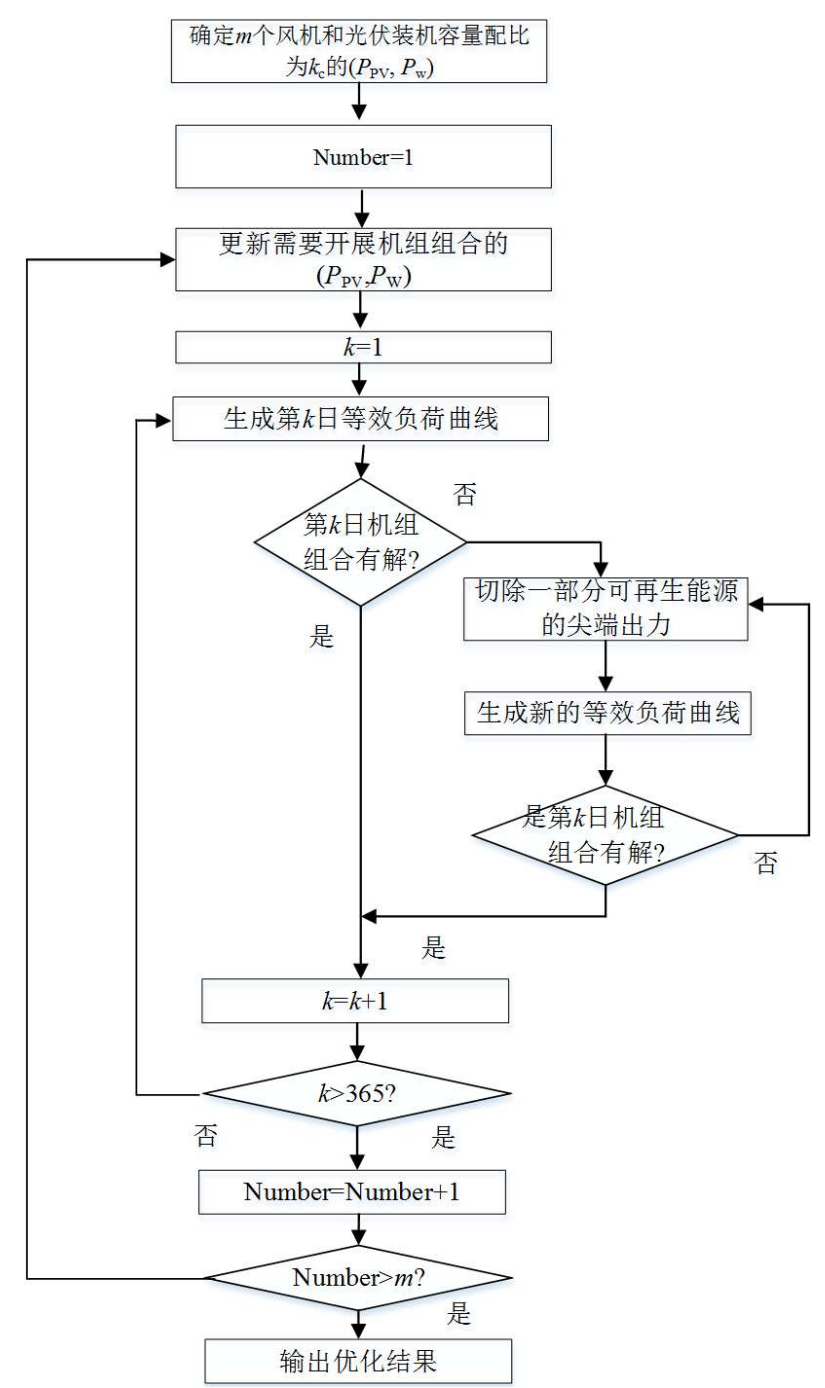

图3 风机光伏装机容量配比为 $k_{\mathrm{c}}$ 时优化模型的求解流程图。

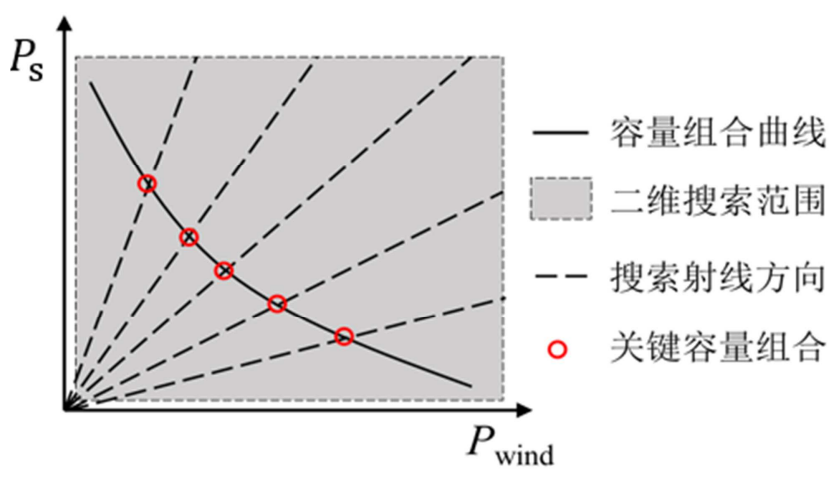

图4 风机和光伏装机容量不同配比曲线图。

\section{4. 算例分析}

对我国某风光较丰富地区开展风光机组装机容量优 化规划。其中火电机组共计 960 万 $\mathrm{kW}$, 包括6台额定容量 60 万 $\mathrm{kW}$ 的超临界机组和 6 台额定容量为 100 万 $\mathrm{kW}$ 的超超 临界机组; 风机和光伏设备的年利用小时数分别为 2133 小时和1490小时, 采用改进的IEEE RTS系统8760 小时负 
荷数据 (冬季与夏季的负荷对调, 以符合中国的负荷特征), 年最大负荷为 800 万 $\mathrm{kW}$ 。

当风机光伏装机容量比值为 $1: 1$ 时, $R=20 \%, C_{\text {coal }}$ 取 600 元/tce时, 可得到表1中的评估结果。由表1可知, 随着风 机和光伏装机容量的增大, 由于可再生能源出力替代了火 电机组, 系统的运行成本逐渐减小, 但是弃风弃光比例也 随之增大。

表1 不同风机和光伏装机容量组合对应的 $C_{2}$ 的评估结果。

\begin{tabular}{llll}
\hline $\boldsymbol{P}_{\mathbf{w}} / \mathbf{M W}$ & $\boldsymbol{P}_{\mathbf{P V}} / \mathbf{M W}$ & $\boldsymbol{R}_{\text {rej }} / \%$ & $\boldsymbol{C}_{2} / \mathbf{y u a n}$ \\
\hline 2350 & 2350 & 1.14 & $7.24 \mathrm{E}+09$ \\
2600 & 2600 & 2.01 & $7.12 \mathrm{E}+09$ \\
2750 & 2750 & 2.99 & $7.09 \mathrm{E}+09$ \\
2900 & 2900 & 3.93 & $7.01 \mathrm{E}+09$ \\
3050 & 3050 & 5.07 & $6.96 \mathrm{E}+09$ \\
3200 & 3200 & 6.07 & $6.92 \mathrm{E}+09$ \\
3300 & 3300 & 6.95 & $6.88 \mathrm{E}+09$ \\
3450 & 3450 & 8.08 & $6.82 \mathrm{E}+09$ \\
3560 & 3560 & 8.72 & $6.81 \mathrm{E}+09$ \\
3600 & 3600 & 9.33 & $6.81 \mathrm{E}+09$ \\
3700 & 3700 & 9.82 & $6.79 \mathrm{E}+09$ \\
3720 & 3720 & 10.01 & $6.77 \mathrm{E}+09$ \\
3800 & 3800 & 10.54 & $6.73 \mathrm{E}+09$ \\
4000 & 4000 & 12.26 & $6.67 \mathrm{E}+09$ \\
4200 & 4200 & 14.11 & $6.62 \mathrm{E}+09$ \\
4400 & 4400 & 15.76 & $6.57 \mathrm{E}+09$ \\
4500 & 4500 & 16.65 & $6.58 \mathrm{E}+09$ \\
4600 & 4600 & 17.55 & $6.56 \mathrm{E}+09$ \\
4800 & 4800 & 18.99 & $6.53 \mathrm{E}+09$ \\
5000 & 5000 & 20.76 & $6.45 \mathrm{E}+09$ \\
\hline
\end{tabular}

如果供电成本中同时考虑 $C_{1} 、 C_{2}$ 和 $C_{3}$, 其中 $C_{\text {un } \_ \text {w }}$ 和

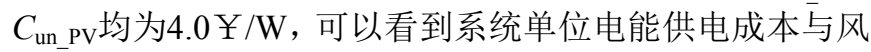
机/光伏装机容量的函数关系如图 5 所示, 此时极值点处对 应的最佳风光机组装机容量组合为 (2860MW,2860MW), 最佳弃风弃光比例为 $3.68 \%$ 。

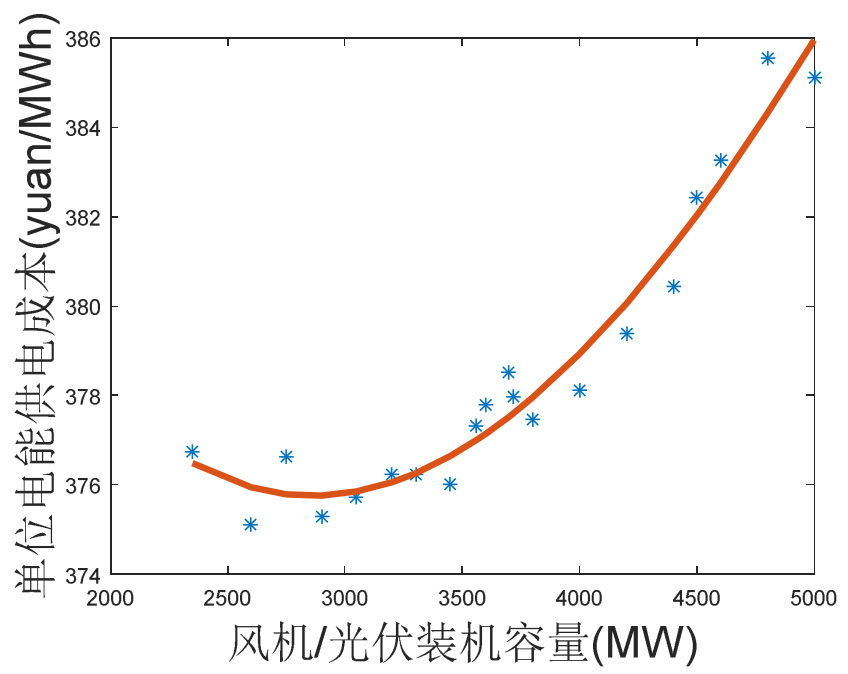

图5 年供电成本与风机/光伏装机容量之间的关系。

考虑到风机和光伏设备成本的下降趋势, 对 $C_{\mathrm{un}}{ }_{\mathrm{w}}$ 和 $C_{\text {un P }}$ 的取值由 $4.0 Y / \mathrm{W}$ 逐渐下降到 $3.6 Y / \mathrm{W} 、 2.8 Y / \mathrm{W}$ 和 2.4 $¥ / \mathrm{W}$, 采用多项式函数拟合 $C_{1}+C_{2}+C_{3}$, 得到图6所示的曲 线, 对应以上拟合函数的极值点分别为: $2860 \mathrm{MW}$, $3188 \mathrm{MW} ， 3549 \mathrm{MW}$ 以及3951MW，对应的弃风弃光比例 分别为 $3.68 \%, 5.99 \%, 8.66 \%$ 以及 $11.84 \%$, 随着风机和光
伏单位投资成本的下降, 最佳风机光伏装机容量组合逐渐 增大, 预示着未来大力发展可再生能源将会是最为经济的 选择。

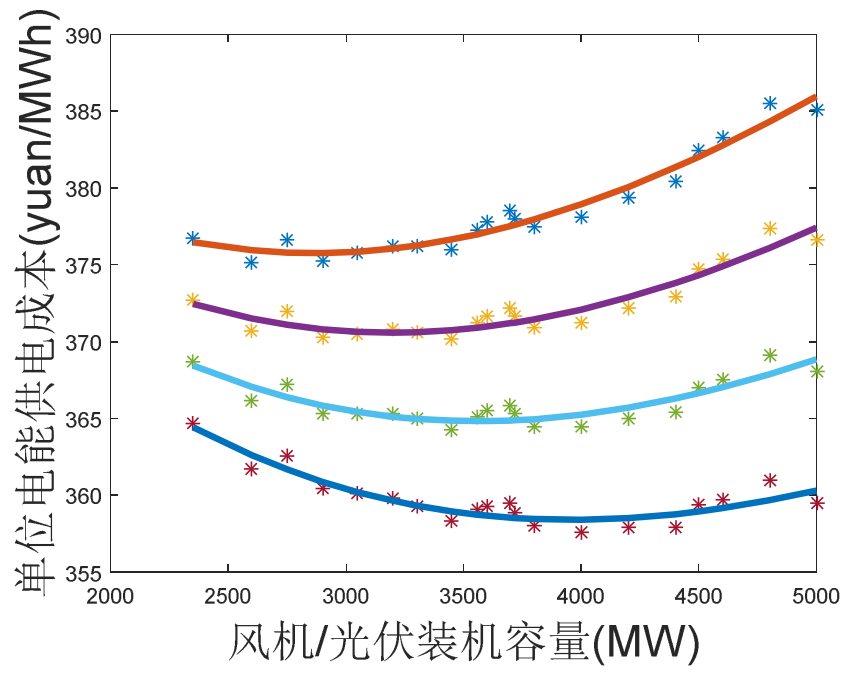

图6 单位电能供电成本随可再生能源单位投资成本变化图。

近年来, 人们的环保意识逐渐增强, 环境成本将会逐 步增加, 随着环境成本的逐渐增加, 系统单位电能供电成 本与风机/光伏装机容量的函数关系如图7所示, 大力发展 清洁的风光电能更加经济环保。

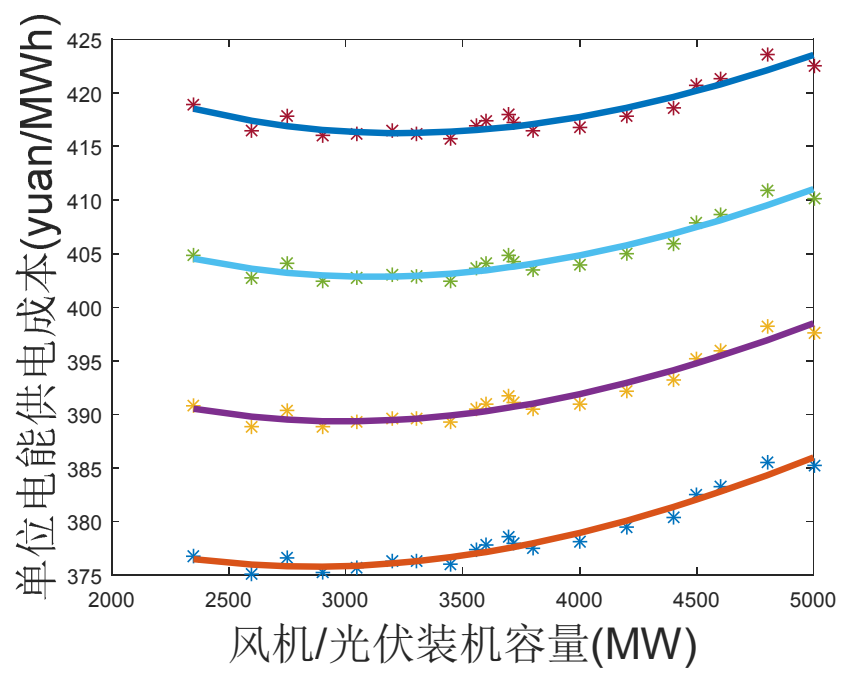

图7 单位电能供电成本随环境成本变化图。

\section{5. 结论}

本文提出了一种基于日前经济调度的风光机组装机 容量的优化规划方法, 允许合理弃置一部分风光机组的尖 峰出力, 从而降低系统面临的调峰等辅助服务方面的成本, 提高系统的供电经济性, 进而确定使得系统供电成本最低 的风机光伏装机容量组合, 通过对供电成本与装机容量开 展函数拟合可求得经济性最佳的风光机组装机容量组合, 由于本文所提优化方法基于日前经济调度仿真, 因此具有 较好的工程价值。此外, 还对风光设备单位投资成本以及 环境成本进行了敏感性分析, 分析各项成本对最佳弃风弃 
光比例的影响趋势, 发现随着风机和光伏单位投资成本的 下降以及环境成本的升高, 最佳风机和光伏装机容量逐渐 增大, 预示着未来大力发展可再生能源将会是最为经济的 选择。

\section{致谢}

本文为中国工程院院地合作项目 (2020HENZDA02) 和第67批博士后基金的阶段性成果之一。

\section{参考文献}

[1] 国家能源局. 2019年光伏发电并网运行情况 [OE/BL]. http://www.nea.gov.cn/2020-02/28/c_138827923.htm.

[2]国家能源局. 2019 年风电并网运行情况 [OE/BL]. http://www.nea.gov.cn/2020-02/28/c_138827910.htm.

[3] 余贻金知. 智能电网基本理念与关键技术 $[\mathrm{M}]$. 科学出版社, 北京, 2019 .

[4] Sun B, Yu Y, Qin C. Should China focus on the distributed development of wind and solar photovoltaic power generation? A comparative study[J]. Applied Energy, 2017, 185:421-439.

[5] Kalaitzakis P K. Methodology for optimal sizing of stand-alone photovoltaic/wind-generator systems using genetic algorithms[J]. Solar Energy, 2006, 80:1072-1088.

[6] José L. Bernal-Agustín, Rodolfo Dufo-López. Multi-objective design and control of hybrid systems minimizing costs and unmet load[J]. Electric Power Systems Research, 2009, 79(1):170-180.

[7] Ruoli T, Xin L, Jingang L. A novel optimal energy-management strategy for a maritime hybrid energy system based on large-scale global optimization[J]. Applied Energy, 2018, 228:254-264.
[8] Ghorbani N, Kasaeian A, Toopshekan A, et al. Optimizing a hybrid wind-PV-battery system using GA-PSO and MOPSO for reducing cost and increasing reliability[J]. Energy, 2017, 154:581-591.

[9] Li Y, Liang W, Tan R. Optimal design of installation capacity and operation strategy for distributed energy system[J]. Applied Thermal Engineering, 2017, 125:756-766.

[10] Li X, Peng Y, Wang W, et al. A method for optimizing installation capacity and operation strategy of a hybrid renewable energy system with offshore wind energy for a green container terminal[J]. Ocean Engineering, 2019, 186:106125.

[11] Ogunjuyigbe A S O, Ayodele T R, Akinola O A. Optimal allocation and sizing of PV/Wind/Split-diesel/Battery hybrid energy system for minimizing life cycle cost, carbon emission and dump energy of remote residential building $[\mathrm{J}]$. Applied Energy, 2016, 171(Jun.1):153-171.

[12] Yang D, Jing Y Q, Wang C, et al. Analysis of renewable energy subsidy in China under uncertainty: Feed-in tariff vs. renewable portfolio standard[J]. Energy Strategy Reviews, 2021, 34(1):100628.

[13] A T C, B L S, C P A. Modeling constraints to distributed generation solar photovoltaic capacity installation in the US Midwest[J]. Applied Energy, 2018, 210:1037-1050.

[14] Olson A, Jones R A, Hart E, et al. Renewable Curtailment as a Power System Flexibility Resource[J]. Electricity Journal, 2014, 27(9):49-61.

[15] Wu O Q, Kapuscinski R. Curtailing Intermittent Generation in Electrical Systems[J]. Manufacturing \& Service Operations Management, 2013, 15(4):578-595.

[16] Wang B, Zhou M, Xin B, et al. Analysis of operation cost and wind curtailment using multi-objective unit commitment with battery energy storage[J]. Energy, 2019, 178(JUL.1):101-114.

[17] Asfaw S A, Bogdanov D, Breyer C. Curtailment-storage-penetration nexus in the energy transition[J]. Applied Energy, 2018, 235:1351-1368. 